\title{
Recyclage des déchets et dynamiques sociales dans la transition du rural au périurbain à la Réunion
}

\author{
Hélène Jarousseau ${ }^{1,2,{ }^{*}}$, Tom Wassenaar ${ }^{3}$, Bathilde Sallote ${ }^{1}$ et Jean-Marie Paillat ${ }^{3}$ \\ ${ }^{1}$ Université catholique de l'Ouest, 3, place André-Leroy, 49008 Angers cedex, France \\ 2 CNRS, UMR 6590 Espaces et sociétés (ESO-Angers), 5 bis, boulevard Lavoisier, 49045 Angers cedex 1, France \\ ${ }^{3}$ CIRAD, UR Recyclage et risque, avenue Agropolis, 34398 Montpellier cedex 5, France
}

\begin{abstract}
Résumé - Dans un contexte de forte urbanisation, la situation insulaire de la Réunion rend complexe la gestion des déchets et incite à trouver des solutions de valorisation. L'épandage des boues de station d'épuration des eaux usées sur la canne à sucre fait l'objet de plusieurs demandes d'autorisation qui sont sources de conflits. L'enjeu d'une plus forte territorialisation des activités agricoles, dont témoigne l'épandage des déchets urbains, perturbe aujourd'hui le rapport entre producteurs et usinier. Cet enjeu différencie nettement le niveau local (agriculteurs, acteurs communaux) du niveau régional (filières, profession agricole, administrations) en termes de prises de position institutionnelles. Ce processus de territorialisation n'est pas nouveau, car il s'inscrit dans des transformations beaucoup plus globales de la société, amorcées avec la départementalisation de l'île en 1946. Sur le plan agricole et sous l'impulsion de la réforme foncière des années 1960, ces transformations se sont d'abord traduites par l'émergence d'une agriculture familiale directement liée à la filière agro-industrielle sucrière au détriment de la grande propriété foncière. Plus récemment, la montée en puissance des enjeux territoriaux et environnementaux et leur prise en compte par les agriculteurs témoignent de la capacité de la société réunionnaise à s'affranchir progressivement de ses anciennes logiques.
\end{abstract}

Mots clés : Réunion (Île de la) / canne à sucre / recyclage des déchets / territoire / société

Abstract - Waste recycling and social dynamics in the transition from rural to the peri-urban in Reunion Island. In a context of rapid urbanization, the insularity of Reunion Island makes waste management complex and calls for finding recycling solutions. Official requests for authorizing the spreading of sewage sludge on sugarcane are a source of conflict. The concern of a stronger territorial integration of agricultural activities, as illustrated by the spreading of urban waste, now disturbs the relationship between sugarcane producers and the sugar industry. This issue clearly differentiates the local (farmers, actors of the municipalities) and regional (agricultural production sectors, agricultural profession, administrations) institutional positions. This process of territorial integration is not new because it is part of the much broader changes in the society, initiated with the departmentalization of the island in 1946. In agriculture, following the land reform of the 1960s, these transformations first resulted in the emergence of a family farming directly bound to the agroindustrial sugar sector, at the expense of large landownership. Then, from the sugar sector logic to today's territorial and environmental issues seized by farmers, this new agriculture of Reunion Island demonstrates the ability of the society to reformulate its concerns by gradually replacing obsolete frameworks.

Keywords: Reunion Island / sugar cane / waste management / territory / society

\section{Introduction}

Dans les années 1980, les boues de station d'épuration des eaux usées (STEU) étaient utilisées comme matières fertili-

\footnotetext{
$\overline{\text { * Auteur de correspondance }}:$ hjarouss@uco.fr
}

santes par les agriculteurs proches des grandes villes. À la fin des années 1990, les lois sur l'eau modifient leur statut de boues pour devenir des déchets (Nicourt et Girault, 2003). Ce statut les introduit dans une problématique du risque et induit dans les représentations ordinaires le rejet des boues urbaines mais aussi la méfiance dans leur utilisation agricole (Barbier et Lupton, 2003). En France, en 2007, seulement $50 \%$ des boues de 
station d'épuration après séchage et chaulage et $10 \%$ après compostage sont recyclées en agriculture (Papy et al., 2012). Le problème de la gestion de ces déchets est exacerbé dans le contexte de la Réunion, département français d'outre-mer d'une superficie de $2500 \mathrm{~km}^{2}$ (Fig. 1) et comptant 835103 habitants (Insee, 2013). Situation insulaire, forte croissance démographique, pression foncière, conditions pédoclimatiques difficiles, concurrence entre déchets et importation coûteuse des engrais rendent particulièrement complexe la recherche de solutions pour la valorisation. Dans la plupart des communes de l'île, les boues de station d'épuration vont toujours en centre d'enfouissement technique (CET). Ces derniers arrivent à saturation et l'enfouissement n'est autorisé qu'à titre dérogatoire. Depuis 2010, avec la mise aux normes des stations d'épuration (Office de l'eau, 2016), la plupart des communes ont soumis des plans d'épandage à l'autorisation préfectorale. En l'absence d'alternatives comme l'incinération ou la transformation en produits homologués ou normés et sous l'injonction européenne et de l'État pour supprimer la décharge en CET, ces plans d'épandage sont progressivement adoptés. Des réserves ont cependant parfois été formulées. Ainsi, l'épandage de boues urbaines sur les cultures cannières, pas encore pratiqué à la Réunion, présente un caractère innovant et suscite, comme ailleurs, la controverse (Barbier et Lupton, 2003). Cette nouvelle dynamique territoriale, liée aux activités urbaines, perturbe le modèle agro-industriel sucrier fondé sur l'agriculture familiale et l'usine, et témoigne d'une agriculture cannière et sucrière plus complexe aujourd'hui. Elle met aussi en exergue la transition du rural vers le périurbain amorcée depuis une quinzaine d'années avec l'urbanisation croissante de l'île.

L'objectif de cet article est de montrer que la question de la valorisation des boues, au-delà de la controverse qu'elle suscite, relève de la territorialisation de l'activité agricole cannière. Trois études de cas de plan d'épandage des boues seront mobilisées, mettant en évidence une disjonction entre une logique filière et l'intérêt territorial. Pour mieux saisir les facteurs de la territorialisation en cours, des travaux relatifs à l'agriculture cannière et portant sur le processus de formation de la société réunionnaise seront pris en compte.

\section{Méthodologie}

Pour traiter cette problématique, nous nous appuyons dans un premier temps sur trois études de cas (Fig. 1) à des niveaux d'organisation territoriale croissants.

\subsection{Au niveau communal}

En 2010, l'équipe municipale de la commune de Bras Panon a choisi de valoriser les boues de STEU par leur épandage sur les cultures cannières proches de la STEU. L'enquête, réalisée dans le cadre d'un master de sociologie en 2010, s'est basée sur des entretiens menés auprès des sept agriculteurs impliqués dans le projet d'épandage des boues, du responsable des services techniques de la commune et du gérant de la station d'épuration. Des entretiens ont aussi été réalisés avec des représentants de l'usine sucrière de Bois Rouge, du syndicat des fabricants de sucre, de la Chambre d'agriculture, du conseil général de la Réunion, et de l'aménagement du territoire panonnais. Des employés ainsi que le directeur d'une coopérative d'approvisionnement en engrais ont également été interviewés.

\subsection{Au niveau intercommunal}

Le projet « Gestion intégrée des résidus organiques par la valorisation agronomique à la Réunion »(Girovar) a mis en œuvre une démarche de co-construction impliquant l'ensemble des acteurs pour explorer le potentiel de valorisation agronomique de tous les résidus organiques disponibles sur le territoire de la côte ouest (TCO) regroupant les communes de La Possession, Le Port, Saint-Paul, Trois-Bassins et Saint-Leu. La concertation s'est articulée entre trois arènes de discussion : représentants institutionnels, experts techniques et « professionnels » regroupant les représentants de groupes cibles (Wassenaar et al., 2015). La question de la valorisation des boues a ainsi été abordée dans cette concertation globale entre monde urbain et monde agricole. Les résultats des réunions et ateliers ont été consignés dans des comptes rendus du projet.

\subsection{Au niveau régional}

Le recueil d'informations sur le plan d'épandage des boues de la station du Grand Prado (communauté de communes de Saint-Denis, Sainte-Marie et Sainte-Suzanne ; Fig. 2) s'est focalisé sur les accords partenariaux entre les collectivités et le monde agricole (protocole de partenariat relatif à la valorisation agricole par épandage des boues de la station d'épuration du Grand Prado, signé le 7 décembre 2009), des articles de presse mettant en exergue les éléments de la controverse, et sur divers documents administratifs (notes, rapport du commissaire enquêteur, arrêté $n^{\circ} 14-2919 / \mathrm{SG} / \mathrm{DRCTCV}$ du 27 février 2014).

Dans un second temps, nous mobilisons des travaux s'intéressant aux transformations de la société réunionnaise opérées depuis la départementalisation de l'île en 1946 et la régionalisation en 1982, qui témoignent d'enjeux pour le développement des localités issues de la réforme foncière (Paillat-Jarousseau, 2001, 2005, 2014).

\section{3 Émergence de projets de valorisation de boues urbaines controversés}

Depuis le début des années 2000, l'urbanisation croissante et la prise en compte des questions environnementales placent les agriculteurs, parmi tous les acteurs, dans les enjeux territoriaux portés par les collectivités (Smith et al., 2001).

À Bras Panon, la surface cannière retenue pour l'épandage des boues urbaines devrait atteindre 56 ha à terme, soit une majeure partie des 100 ha disponibles pour l'épandage à proximité de la station d'épuration. La réglementation sur les boues urbaines interdisait leur épandage à la Réunion, en raison de teneurs élevées en éléments traces métalliques (ETM) des sols, ceux-ci dépassant les seuils réglementaires pour le nickel, le chrome et le cuivre (décret $n^{\circ}$ 97/1133 du 12 août 1997 et arrêté supplémentaire du $1^{\text {er }}$ août 1998). Une dérogation a été rendue possible par les travaux de recherche de Collin et Doelsch (2010), qui ont montré l'origine naturelle des ETM 


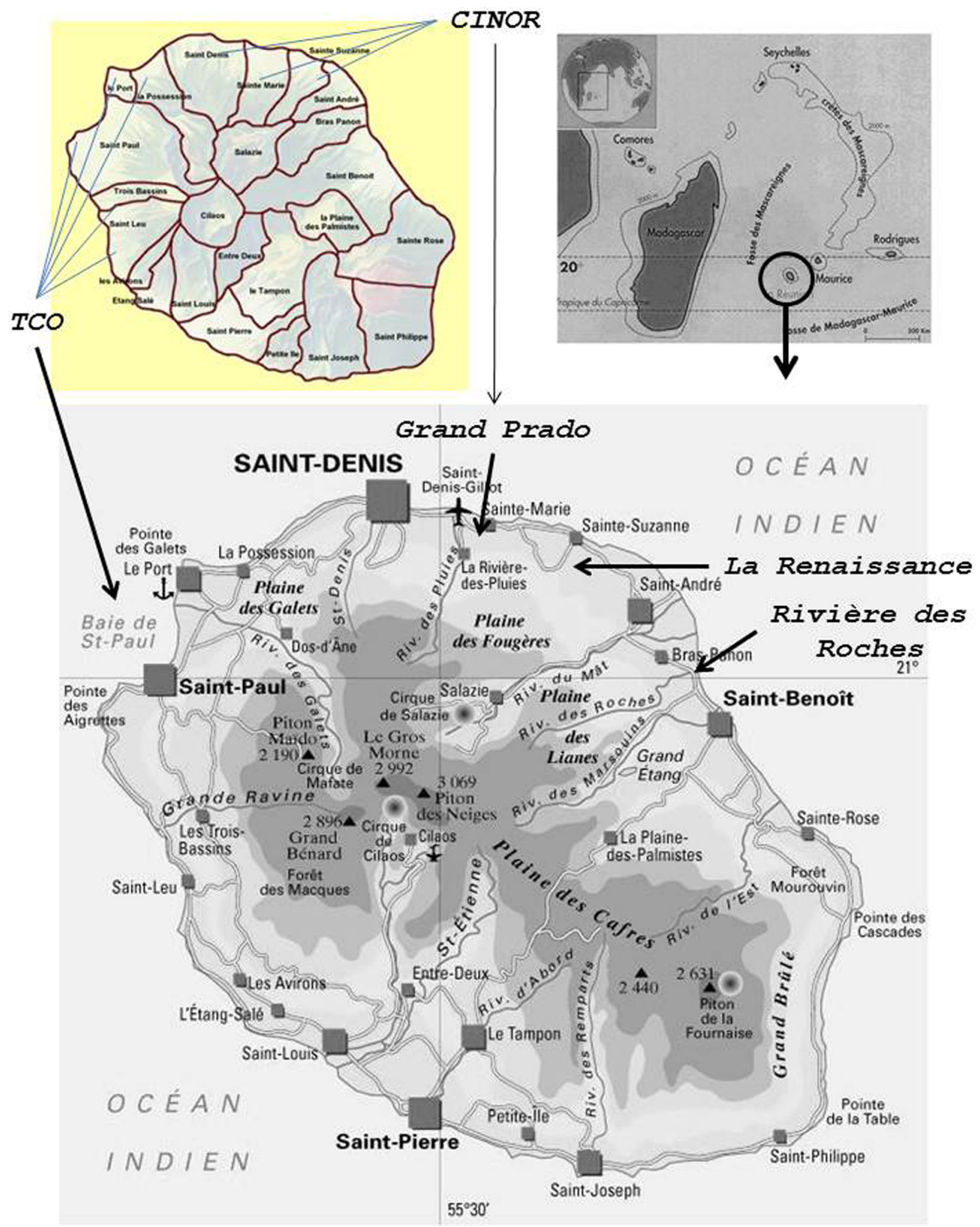

Fig. 1. Situation de la Réunion, département français d'outre-mer dans l'océan Indien, et positionnement des cas d'étude : Rivière des Roches (commune de Bras Panon), communautés de communes CINOR (communauté intercommunale du Nord) et TCO (territoire de la côte ouest) ainsi que la localité de La Renaissance ayant fait l'objet d'une étude ethnographique approfondie.

Fig. 1. Situation of Reunion Island, French overseas department in the Indian Ocean and position of case studies: Rivière des Roches (Bras Panon municipality), municipalities associations of CINOR (communauté intercommunale du Nord) and TCO (territoire de la côte ouest) and the locality of La Renaissance, which has been studied by anthropologist. 


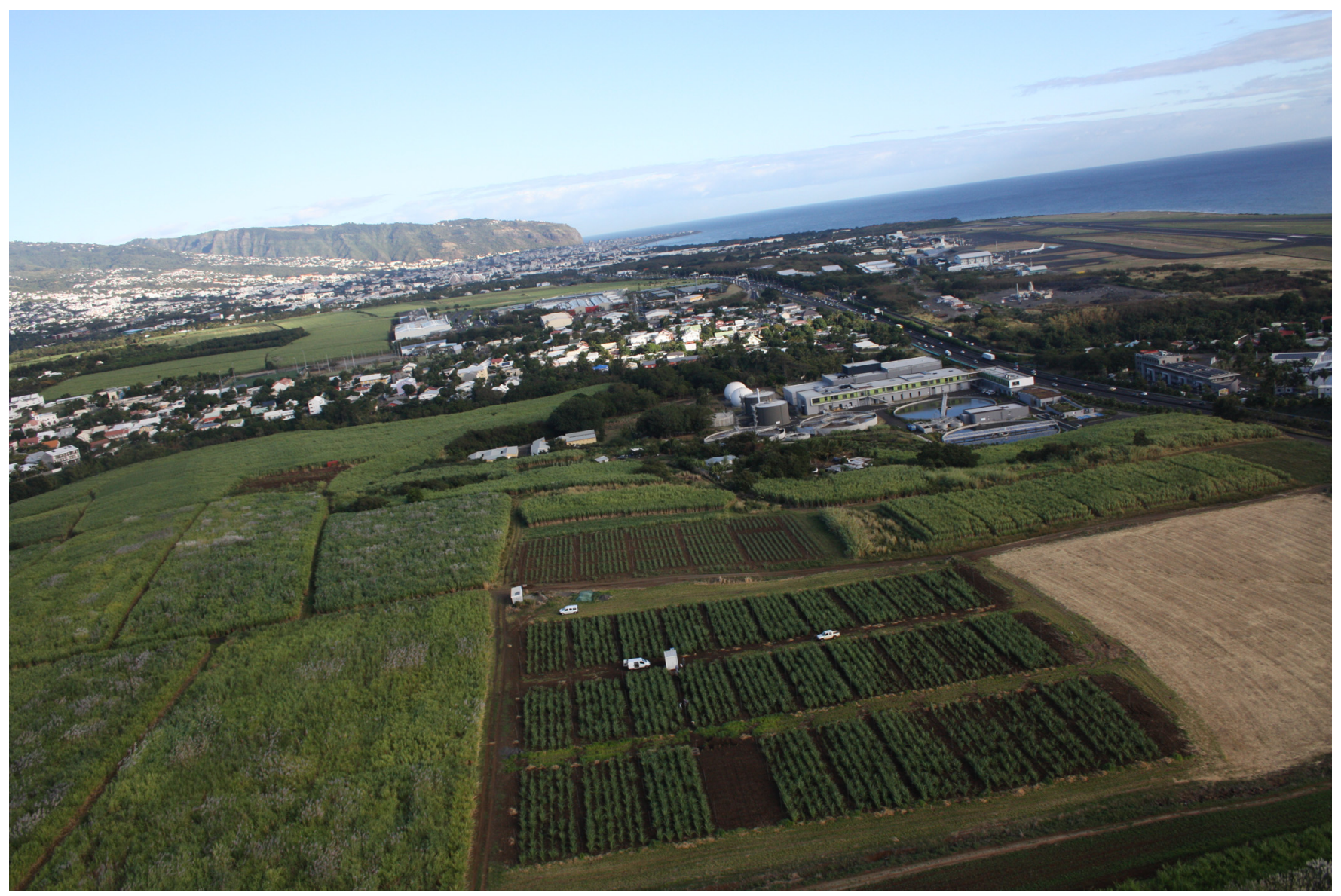

Fig. 2. Vue du dispositif expérimental CIRAD (centre de recherche) et de la station d'épuration du Grand Prado (au premier plan), des zones urbanisées proches de l'aéroport à Sainte-Marie (second plan) et de la ville de Saint-Denis (en arrière-plan) (crédit : C. Poser, CIRAD).

Fig. 2. View of the CIRAD (research centre) experimental device and Grand Prado waste water plant (at the forefront), urban areas near airport of Sainte-Marie and Saint-Denis town (background).

dans les sols d'origine volcanique, leur faible mobilité et leur faible phyto-disponibilité. À l'instar de celui de Bras Panon, d'autres projets de plans d'épandage voient le jour, notamment ceux concernant les grandes communautés d'agglomérations impactant des surfaces dépassant le millier d'hectares.

Le projet de Bras Panon a été bien accueilli localement par les agriculteurs, l'apport de boues se substituant en partie aux engrais minéraux dont les prix ont fortement augmenté depuis quelques années. Outre l'apport d'éléments fertilisants (azote, phosphore), les boues ont par ailleurs un effet d'amendement (calcium, magnésium, matière organique) pour les sols. Selon les agriculteurs enquêtés en 2010, l'apport de boues permettrait une croissance de leur production, un meilleur rendement, une canne plus belle et plus riche en saccharose. Au moment des enquêtes, l'épandage des boues est également paradoxalement bien perçu par certaines coopératives, comme l'explique ce responsable : «Et si je pouvais en vendre moins [des engrais] ou ne plus en vendre, cela arrangerait les comptes de la coopérative [...]. Économiquement aujourd'hui on s'en sortirait mieux si on stoppait la distribution d'engrais. \ Ceci s'explique par le coût élevé des engrais qui ne permet plus aux coopératives de réaliser une marge suffisante à la vente. Ainsi, la mise à disposition des boues urbaines ne déstabilise pas leur équilibre financier et n'entre pas en concurrence de l'engrais devenu peu rémunérateur. Par ailleurs, les coopératives se montraient aussi intéressées par la vente de compost fabriqué à partir de boues urbaines.

L'épandage de boues sur les terres cannières est cependant vu de diverses façons par les parties prenantes selon leurs préoccupations. Pour l'usinier, la mise en place d'un projet d'épandage sous la responsabilité de la mairie présente l'intérêt de ne pas déclasser vers l'habitat les terres cannières dont la production est essentielle pour l'approvisionnement de l'usine. D'un point de vue financier, la mairie a également intérêt à épandre les boues d'épuration plutôt que de payer la mise en CET.

D'autres, les producteurs d'effluents d'élevage, notamment porcins et avicoles, sont opposés à ce type de projet, craignant que des plans d'épandage de boues urbaines concurrencent ceux des effluents d'élevage qui ne sont pas moins importants pour l'environnement. En 2007, la quantité potentielle épandable d'azote, sur laquelle sont souvent basés les plans d'épandage, calculée sur les cheptels animaux et la population raccordable aux stations d'épuration existantes ou en projet, s'élevait à $3046 \mathrm{t}$ par an pour les effluents d'élevage et $363 \mathrm{t}$ par an pour les boues (DAF et CIRAD, 2007). En 2014, l'Office de l'eau (2016) chiffre la production de boues à $6268 \mathrm{t}$ par an de matières sèches, soit très approximativement $275 \mathrm{t}$ 
par an d'azote ; la mise aux normes des stations se poursuivant, ce chiffre se rapproche du potentiel calculé de 363 t d'azote par an. La concurrence paraît déloyale pour la Fédération régionale des coopératives agricoles (FRCA), car les éleveurs ont besoin de cultures cannières et maraîchères pour épandre leurs effluents. Pour la FRCA, sur un plan sanitaire, un protocole expérimental ou de suivi à long terme sur les sols, la canne, le jus de canne et le sucre devrait être mis en œuvre avant d'autoriser l'épandage de boues urbaines à la Réunion.

Face à un tel projet, l'usinier craint aussi quant à lui qu'il impacte négativement l'image du sucre réunionnais et s'inquiète des effets sur la vente du sucre et produits dérivés. Alors que des boues simplement séchées seront épandues à Bras Panon (articles dans Le Quotidien de la Réunion, 26 janvier 2016, et Le Journal de l'île, 17 février 2016), l'usinier préférerait qu'elles soient transformées en compost pour les hygiéniser. Cependant, selon le responsable des services techniques de la mairie, entre la préservation des terres à canne et la garantie de boues bien traitées et utilisées, l'usinier ne constitue pas un réel obstacle. Mais, si la concertation entre acteurs à l'échelle communale a été rendue possible par la relative exiguïté du plan d'épandage de boues, il n'en a pas été de même dans d'autres régions de l'île.

Dans le projet Girovar (Wassenaar et al., 2015), des scénarios de valorisation agricole des produits résiduaires aussi bien agricoles qu'agro-industriels et urbains ont été co-construits avec les acteurs. La promesse initiale ayant permis l'adhésion des acteurs était que l'exploitation de la diversité de résidus organiques permettrait de mettre au point des produits fertilisants bien adaptés aux besoins des agriculteurs du territoire. Cela semblait un paradigme propice à la dissipation de la tension entre « mondes » urbains et agricoles entretenue par la logique de compétition entre déchets. Dans l'arène des experts, les groupes de travail ont élaboré un scénario de production d'engrais organique à partir d'un co-compost de boues et déchets verts. Un tel scénario apparaissait pertinent sur les plans technique, agronomique et, surtout, économique, avec un impact positif sur la rentabilité d'une telle filière. La consultation des professionnels n'a pas rencontré d'opposition, du fait du poids du critère prix. C'est finalement au niveau des institutionnels que l'option a été radicalement éliminée. Parmi les experts, les responsables de coopératives agricoles et de l'industrie sucrière se sont ensuite rangés derrière la Chambre d'agriculture représentée ici par ses élus. C'est à ce niveau institutionnel global que perdure la méfiance entre mondes urbains et agricoles encore largement conceptualisés comme distincts et opposés. Bien que déclaré d'intérêt général, le portage du projet par le président du TCO et le sous-préfet de Saint-Paul a pu mettre les responsables agricoles sur la défensive, suspicieux que cet intérêt soit surtout celui de la collectivité.

Le cas du Grand Prado, qui produit depuis fin 2013 des boues digérées, chaulées et séchées sous forme de pellets, a fait émerger des prises de position plus radicales. La Chambre d'agriculture et le Syndicat du sucre, ayant pourtant signé un protocole d'accord pour la valorisation de ces boues en agriculture dès 2009, se sont opposés en 2013 au plan d'épandage du Grand Prado et sont dorénavant contre toute valorisation agricole des boues urbaines à la Réunion (articles dans Le Journal de l'île, 29 août 2013, et Le Quotidien de la Réunion, 26 mai 2014; lettre du Syndicat du sucre au commissaire enquêteur du 21 août 2013). Les arguments développés concernent la concurrence des plans d'épandage d'effluents d'élevage, le problème de contaminants spécifiques aux boues et l'image commerciale du sucre et des produits agricoles en général (article dans Le Journal de l'île, 27 mai 2013). L'administration rend des avis partagés, favorables à l'épandage des boues pour la direction de l'environnement et défavorables pour la direction de l'agriculture. Cette position de l'administration agricole n'a pas été constante, puisque en 2010, la direction de l'agriculture avait incité la profession agricole à la valorisation des boues par épandage sur canne à sucre. Malgré cette opposition, au vu des résultats d'enquête publique, le préfet de la Réunion a autorisé le plan d'épandage du Grand Prado, sous réserve de mettre en place une étude visant à mesurer l'apport potentiel de contaminants par les boues dans la chaîne d'extraction du sucre.

Dans ce contexte, deux dispositifs expérimentaux agronomiques ont été mis en place. Le Système d'Observation et d'Expérimentation au long terme pour la Recherche en Environnement : concernant le recyclage des Produits Résiduaires Organiques (SOERE-PRO) (Houot, 2011) est un dispositif national dont un des sites a été installé en 2013 à la Réunion par le CIRAD (article dans Le Quotidien de la Réunion, 26 mai 2014). Son objectif est d'étudier l'effet du recyclage de PRO (boues de STEU, lisier de porc, fumier de volailles) sur la qualité des sols, des plantes récoltées, des eaux et sur les émissions gazeuses générées par les épandages. La seconde expérimentation, installée en 2014 par eRcane (institut R\&D de la filière canne), vise à quantifier l'intérêt des apports organiques (écumes de sucrerie, effluents d'élevage, boues urbaines, composts) pour fertiliser la canne à sucre.

Aujourd'hui, la préfecture de la Réunion (note du préfet de la Réunion - DEAL - service de l'eau du 12 mai 2016) relance les discussions et va rendre exécutoire l'arrêté pour revenir dans la légalité vis-à-vis des concessionnaires gérant les eaux usées. Le Syndicat du sucre ne s'oppose plus aussi catégoriquement à l'épandage dès lors qu'il s'agirait de produits homologués ou normés ; les boues ne seraient plus alors soumises aux plans d'épandage car sortant réglementairement du statut de déchet. Dans ce nouveau contexte, les essais agronomiques mis en œuvre par la recherche présentent un regain d'intérêt pour l'ensemble des acteurs.

Ainsi, la Réunion n'échappe pas à la permanence des conflits qui accompagne l'histoire du traitement des eaux usées et du recyclage agricole des résidus produits (Nicourt et Girault, 2003 ; Joncoux, 2013 ; Queste, 2016). Avec la controverse sur l'épandage des boues urbaines, on assiste actuellement à une coalition entre professionnels de l'agriculture pour s'opposer à la valorisation agricole de résidus urbains. Mais ce mouvement n'est pas unanime puisque la position des représentants des agriculteurs diffère de celles des agriculteurs qui peuvent être, localement, favorables aux différents projets.

\section{4 Évolution du modèle agro-industriel sucrier née de la transformation de la société réunionnaise}

Les controverses sur les boues sont bien documentées par les sociologues (Barbier et Lupton, 2003 ; Nicourt et Girault, 2003) dans le contexte français et elles ne semblent pas différer fondamentalement dans le contexte réunionnais. Notre propos 
n'est donc pas ici de discuter ou de compléter cette connaissance, la question des boues étant trop récente à la Réunion. Par contre, les dynamiques sociales engendrées par cette problématique révèlent des transformations beaucoup plus profondes de la société réunionnaise inscrite dans un passé original, celui de la plantation. Ainsi, la valorisation des boues urbaines par l'agriculture cannière n'est qu'un élément supplémentaire parmi de nombreux autres, caractérisant le passage d'une société fortement hiérarchisée et verticale du temps de la plantation à une société plus ouverte et horizontale avec un accès de la population à différents niveaux d'intégration, depuis le local de l'activité agricole jusqu'au global des institutions.

La Réunion est en effet une ancienne colonie française où la société et l'économie des plantations sucrières (Mintz, 1986) dominèrent l'île de 1815 jusqu'au début des années 1980 (Combeau et al., 2002 ; Vaxelaire, 2009). Durant cette longue période, les grands propriétaires contrôlaient en amont le procès social de production de la canne en organisant le travail de la main-d'œuvre au champ ; en aval, ils possédaient l'outil industriel de transformation de la canne en sucre. Dans le cadre de la départementalisation, intervenue en 1946, la société réunionnaise est entrée dans un vaste programme de reconstruction d'après-guerre (Combeau et al., 2002 ; Vaxelaire, 2009).

Sur le plan agricole, la réforme foncière a permis de résoudre les difficultés économiques des grands domaines sucriers, confrontés dès les années 1950 au renchérissement de la main-d'œuvre et aux difficultés de mise en valeur des terres (mécanisation difficile). Les terres redistribuées ont été vendues aux travailleurs des grands domaines pour constituer des exploitations familiales de 5 à 10 ha. En 1994, $50 \%$ des livraisons de canne à sucre à l'usine étaient effectuées par les nouveaux exploitants (Chastel, 1995). Depuis la fin des restructurations d'usines en 1996 et le retrait des grands propriétaires de l'industrie sucrière, deux usines, Le Gol dans le Sud de l'île et Bois Rouge dans l'Est, dirigées par le groupe coopératif TEREOS, assurent aujourd'hui la production sucrière (Fig. 1). Le pouvoir des grands propriétaires s'est ainsi déplacé au profit des usiniers qui contrôlent l'usine et la valorisation des produits sucriers. Celle-ci continue d'être très soutenue par des fonds nationaux et européens dans un contexte de pression accrue sur le foncier (Fusillier et al., 2006). Malgré le recul de la grande propriété, laquelle ne couvrait plus que $13 \%$ de la surface en 2000 pour moins de $0,5 \%$ de l'effectif des exploitants (Fusillier et al., 2006), pour l'agriculteur l'usine demeure l'unique débouché commercial. Les exploitations familiales issues de la réforme constituent le modèle agricole qui perdure, les très petites exploitations ayant pour la plus grande majorité disparu.

Le passage de la grande propriété cannière à l'agriculture familiale s'inscrit dans un mouvement plus vaste de déclin de la plantation et d'émergence de nouvelles formes d'intégration à la société. La société réunionnaise s'est progressivement diversifiée en offrant des emplois en dehors du secteur primaire et en redéfinissant profondément son territoire. Les anciens domaines devenus des quartiers au sein des communes témoignent de la pluralité des modes d'intégration à la société (Paillat-Jarousseau, 2005). Dans le changement induit par la départementalisation, puis la régionalisation en 1982, l'accès aux emplois communaux a permis aux habitants de promouvoir leur lieu de vie. Des associations à vocation sociale, culturelle ou religieuse ont été créées. Ainsi, les habitants ont tissé des liens avec les institutions communales mais aussi le département et la région, entraînant une plus grande autonomie. Ces logiques montrent une évolution de la société qui affranchit progressivement les populations locales des anciens cadres hérités du passé. Peu à peu, la population a surmonté l'ordre colonial en faveur d'une société plus ouverte, offrant de nouvelles possibilités. La société réunionnaise se complexifie, s'urbanise fortement depuis le début des années 2000 et atteste de nouveaux enjeux territoriaux. Le tissu social issu du passé des plantations doit désormais relever les défis d'une société diversifiée par le développement croissant des secteurs d'activités tertiaires et quaternaires.

Dans ce nouveau contexte, le recyclage des déchets urbains, qui lie les exploitants agricoles aux actions des collectivités, rejoint la pluralité des enjeux locaux, en attribuant aux agriculteurs un nouveau rôle en matière de développement durable. Les exploitants ne sont plus uniquement producteurs de canne à sucre mais également acteurs de la vie écologique et pourvoyeurs de services environnementaux. Sur le plan de la structure des sociétés locales, l'urbain constitue une nouvelle opportunité tout en témoignant d'un enjeu agricole qui repose sur les mêmes bases sociales. Les configurations sociales, observées 20 ans après la réforme (Paillat-Jarousseau, 2014), permettent aux producteurs de canne à sucre de jouer aujourd'hui un rôle actif dans la construction des territoires, en devenant des partenaires de projets territoriaux.

\section{Conclusion}

L'histoire de la Réunion et de sa production cannière témoigne de mutations majeures sur ce territoire insulaire. Société fondée sur des rapports sociaux inégaux et hiérarchiques entre une minorité de grands propriétaires fonciers et une majorité de travailleurs, cet univers contraignant a façonné de façon profonde la société réunionnaise. Dans le contexte de la départementalisation, la réforme foncière de la fin des années 1960 a permis l'émergence d'une nouvelle forme sociale de production de la canne à sucre marquant la séparation de l'amont et de l'aval de la filière sucrière vers davantage d'autonomie des producteurs de canne à sucre. En s'affranchissant des multiples dépendances vis-à-vis des grands propriétaires, la société a évolué vers des formes d'intégration où les localités attestent d'enjeux pluriels tout à la fois économiques, sociaux et culturels.

L'urbanisation croissante de la Réunion et de ses territoires, marquant une transition historique du rural au périurbain, est une nouvelle opportunité d'intégration horizontale. L'enjeu agricole repose sur les mêmes bases sociales et demeure fortement déterminé par l'enjeu de filière. Mais, certains producteurs de canne à sucre entendent jouer un rôle actif dans la construction territoriale en devenant des partenaires de projets à cette échelle. Jusque-là, leur identité se limitait essentiellement au rôle économique de production. Dans le cadre de projets tels que l'épandage de déchets urbains, ils se voient attribuer un rôle déterminant et de nouvelles figures se dessinent. Cette situation infléchit les trajectoires professionnelles en les plaçant dans de nouveaux rapports sociaux. Territoires de culture et d'identité, on assiste à la reformulation des territoires canniers en engendrant à 
plus ou moins long terme des effets sur la signification profonde du métier. Mais cette autonomisation est aussi source de conflits et génère des contradictions entre le niveau d'organisation local, des agriculteurs intégrés à leur localité, et le niveau régional où les acteurs urbains (les grandes communautés de communes) et agricoles (les filières de production) se différencient plus nettement et s'opposent. L'avenir de la valorisation des boues urbaines reste donc incertain, notamment concernant l'autorisation de plans d'épandage dans le cadre d'une logique « déchet ». La solution qui semble se dessiner serait d'opter pour une logique " produit » avec l'homologation en cours pour les boues digérées, chaulées, séchées et pelletisées du Grand Prado ou la normalisation NFU 44095 pour les co-composts de boues et déchets verts.

Remerciements. Les études de cas présentés dans cet article ont bénéficié des financements du projet ANR STRA 15-08 ISARD (2009-2013) et du projet CAS-DAR (ministère de l'Agriculture) GIROVAR (2011-2014).

\section{Références}

Barbier R, Lupton S. 2003. Jeux et enjeux autour de la réglementation des épandages, une analyse socio-économique. Doss Environ INRA 25: 139-148.

Chastel JM. 1995. Le rôle des institutions dans l'évolution de la filière canne à la Réunion. Thèse de doctorat en agro-économie, ENSA (Montpellier), $465 \mathrm{p}$.

Collin B, Doelsch E. 2010. Impact of high natural soil borne heavy metal concentrations on the mobility and phytoavailability of these elements for sugarcane. Geoderma 159(3-4): 452-458.

Combeau Y, Maestri E, Eve P, Fuma S. 2002. Histoire de la Réunion. De la colonie à la région. Paris : Nathan.

Direction de l'agriculture et de la forêt (DAF), Centre de coopération internationale en recherche agronomique pour le développement (CIRAD). 2007. Atlas des matières organiques issues des activités d'élevage et d'assainissement urbain à la Réunion. Saint-Denis, la Réunion : DAF Réunion, CIRAD, $70 \mathrm{p}$.

Fusillier JL, Choisis JP, Piraux M. 2006. Le modèle d'exploitation agricole familiale réunionnais face à l'accroissement des contraintes foncières et économiques. Cah Agric 15(6): 542-548.
Houot S. 2011. SOERE-PRO : impacts environnementaux du recyclage de produits résiduaires organiques sur les écosystèmes cultivés - Labellisation 2011 des systèmes d'observation et d'expérimentation sur le long terme, pour la recherche en environnement. In : Dossier pour la consolidation de la labellisation du SOERE-PRO (2011-2013), 41 p.

Insee. 2013. Évolution de la population totale au 1er janvier 2013. Paris : Institut national de la statistique et des études économiques.

Joncoux S. 2013. L'intensification écologique par la valorisation des déchets organiques : de l'écologisation aux inégalités. Nat Sci Soc 21(2): 223-229.

Mintz S. 1986. Sweetness and power. The place of sugar in modern history. New York: Penguin Books.

Nicourt C, Girault JM. 2003. Qualification des déchets des boues de stations d'épuration et réorganisation de la filière, dans deux départements marqués par le moteur urbain. Doss Environ INRA 25: $125-138$.

Office de l'eau. 2016. Panorama des services publics d'assainissement de la Réunion. Chron Eau Réun 66-4: 15 p.

Paillat-Jarousseau H. 2001. Une terre pour cultiver et habiter. Anthropologie d'une localité de l'Île de la Réunion. Paris : L'Harmattan.

Paillat-Jarousseau H. 2005. The transformation of social relationships in a community as a result of land reform: a case study of a former large sugar cane estate in Reunion. Can Rev Sociol Anthropol 42(1): 93-110.

Paillat-Jarousseau H. 2014. Agriculture familiale sur l'Île de la Réunion. Le rôle des dynamiques sociales dans la réforme foncière. Rev Tiers-Monde 220(1): 81-98.

Papy F, Mathieu N, Ferault C. 2012. De nouveaux rapports à la nature dans les campagnes. Paris : Éditions QUAE.

Queste J. 2016. Concertation et changements. Le cas du recyclage des déchets organiques à la Réunion. Thèse de doctorat de l'université de Grenoble-Alpes, 253 p.

Smith A, Sorbets S, Faure A, Le Bart C, Barraize F. 2001. Leaderships et arrangements territoriaux. Optiques et niveaux d'analyse. Sci Soc 53: 5-83.

Vaxelaire D. 2009. L'histoire de la Réunion. De 1848 à nos jours. 2e éd. Vol 2. La Réunion : Éditions Orphie.

Wassenaar T, Queste J, Paillat JM, Saint Macary H. 2015. La coconstruction de filières de recyclage de résidus organiques à la Réunion. Innov Agron 43: 161-175.

Citation de l'article : Jarousseau H, Wassenaar T, Sallote B, Paillat J-M. 2016. Recyclage des déchets et dynamiques sociales dans la transition du rural au périurbain à la Réunion. Cah. Agric. 25: 65002. 